\title{
BRDOKHTSEN
}

NATIONAL LABORATORY

BNL-90269-2009-CP

\section{Thermal neutron imaging in an active interrogation environment}

\author{
Peter E. Vanier, Leon Forman, and Daren R. Norman
}

Presented at the $20^{\text {th }}$ International AIP Conference

July 2009

\author{
Nonproliferation and National Security Department \\ Brookhaven National Laboratory \\ P.O. Box 5000 \\ Upton, NY 11973-5000 \\ www.bnl.gov
}

\begin{abstract}
Notice: This manuscript has been authored by employees of Brookhaven Science Associates, LLC under Contract No. DE-AC02-98CH10886 with the U.S. Department of Energy. The publisher by accepting the manuscript for publication acknowledges that the United States Government retains a non-exclusive, paid-up, irrevocable, world-wide license to publish or reproduce the published form of this manuscript, or allow others to do so, for United States Government purposes.
\end{abstract}

This preprint is intended for publication in a journal or proceedings. Since changes may be made before publication, it may not be cited or reproduced without the author's permission. 


\section{DISCLAIMER}

This report was prepared as an account of work sponsored by an agency of the United States Government. Neither the United States Government nor any agency thereof, nor any of their employees, nor any of their contractors, subcontractors, or their employees, makes any warranty, express or implied, or assumes any legal liability or responsibility for the accuracy, completeness, or any third party's use or the results of such use of any information, apparatus, product, or process disclosed, or represents that its use would not infringe privately owned rights. Reference herein to any specific commercial product, process, or service by trade name, trademark, manufacturer, or otherwise, does not necessarily constitute or imply its endorsement, recommendation, or favoring by the United States Government or any agency thereof or its contractors or subcontractors. The views and opinions of authors expressed herein do not necessarily state or reflect those of the United States Government or any agency thereof. 


\title{
Thermal neutron imaging in an active interrogation environment
}

\author{
Peter E. Vanier ${ }^{1}$, Leon Forman ${ }^{2}$, and Daren R. Norman ${ }^{3}$ \\ ${ }^{1}$ Brookhaven National Laboratory, Upton, NY 11973, USA \\ ${ }^{2}$ Ion Focus Technology, Inc., Miller Place, NY 11764,USA \\ ${ }^{3}$ Idaho National Laboratory, Idaho Falls, ID 83415, USA
}

\begin{abstract}
We have developed a thermal-neutron coded-aperture imager that reveals the locations of hydrogenous materials from which thermal neutrons are being emitted. This imaging detector can be combined with an accelerator to form an active interrogation system in which fast neutrons are produced in a heavy metal target by means of excitation by high energy photons. The photo-induced neutrons can be either prompt or delayed, depending on whether neutronemitting fission products are generated. Provided that there are hydrogenous materials close to the target, some of the photo-induced neutrons slow down and emerge from the surface at thermal energies. These neutrons can be used to create images that show the location and shape of the thermalizing materials. Analysis of the temporal response of the neutron flux provides information about delayed neutrons from induced fission if there are fissionable materials in the target. The combination of imaging and time-of-flight discrimination helps to improve the signal-to-background ratio. It is also possible to interrogate the target with neutrons, for example using a D-T generator. In this case, an image can be obtained from hydrogenous material in a target without the presence of heavy metal. In addition, if fissionable material is present in the target, probing with fast neutrons can stimulate delayed neutrons from fission, and the imager can detect and locate the object of interest, using appropriate time gating. Operation of this sensitive detection equipment in the vicinity of an accelerator presents a number of challenges, because the accelerator emits electromagnetic interference as well as stray ionizing radiation, which can mask the signals of interest.
\end{abstract}

Keywords: Neutron, imaging, thermalization, helium-3, wire chamber, position-sensitive, coded aperture.

PACS: 25.85.Jg Photofission; 28.41.Rc Instrumentation; 29.40.Gx Position sensitive detectors

\section{INTRODUCTION}

Free neutrons can be produced with accelerators by means of energetic interactions of beams of photons or nucleons on appropriate target nuclei. When first generated, they have kinetic energies typical of fission, alpha, or fusion reactions in the range 1-14 MeV. During the first few hundred microseconds after being liberated, a neutron usually undergoes a series of elastic collisions with surrounding nuclei and transfers its kinetic energy to the environment until it approaches thermal velocities of about $2200 \mathrm{~m} / \mathrm{s}$. Outside of a nucleus, the neutrons have a half-life of about 12 minutes before they decay spontaneously to a proton, an electron and an antineutrino. In air, the scattering mean free path of $2-\mathrm{MeV}$ fission neutrons is about $100 \mathrm{~m}$, while thermal neutrons with mean energy of $0.025 \mathrm{eV}$ have a scattering mean free path of about $20 \mathrm{~m}$. We have developed a coded aperture thermal neutron imaging system that produces images showing the locations from which thermal neutrons are emitted into the air. When combined with an accelerator, the imager improves on a non-imaging active interrogation system by extracting weak signals from statistical fluctuations by means of spatial filtering. In order to be useful for such accelerator applications, the detection system must satisfy certain requirements not needed for passive measurements.

\section{PRIOR WORK}

Coded aperture imaging of thermal neutron sources has previously been proposed for a number of applications, including warhead counting for treaty verification[1], detecting nuclear smuggling[2], explosives detection[3,4], monitoring stored spent nuclear fuel[5]. In earlier testing of our coded aperture camera, which uses a 2-dimensional He-3 positionsensitive wire chamber and a Cd mask, we used isotopic sources (such as ${ }^{252} \mathrm{Cf}$ or ${ }^{241} \mathrm{Am}-\mathrm{Be}$ ) which continuously emitted fast neutrons that were then thermalized in paraffin. We also showed that the 
imager could be operated in a gated mode in combination with a D-T neutron generator to produce images of hydrogenous materials (simulating explosives). In that case[3], the time gate for the data acquisition was chosen to reduce the response of the He-3 chamber to neutrons with energies above the 1$\mathrm{eV}$ absorption threshold of the $\mathrm{Cd}$ mask. Such epithermal neutrons travel about 1.4 meters in $100 \mu \mathrm{s}$ or less, and can be detected by ${ }^{3} \mathrm{He}$ with an efficiency of a few percent. More recent work[6] has made use of pulsed electron accelerators with tungsten targets that produce intense beams of bremsstrahlung photons with energies up to $20 \mathrm{MeV}$. When these energetic photons interact with a heavy metal target (such as $\mathrm{Pb}$, $\mathrm{Bi}, \mathrm{W}$ or $\mathrm{U}$ ) they can excite prompt photoneutrons. In addition, fissionable materials such as $U$ can be excited to fission, producing both prompt and delayed neutrons that continue to be emitted for several seconds after a beam pulse. If the target is surrounded by hydrogenous material, a fraction of the neutrons will be thermalized and can be imaged with the coded aperture. The fissionable material can be distinguished from other heavy metal by adjusting the data acquisition time gate. This type of measurement has previously been carried out using non-imaging PhotoNuclear Detectors (PNDs) consisting of ${ }^{3} \mathrm{He}$ tubes partially moderated with polyethylene and shielded from external thermal neutrons with $\mathrm{Cd}$ sheet around the moderator. The PND is therefore not sensitive to thermal neutrons, and is sometimes operated in combination with a bare ${ }^{3} \mathrm{He}$ tube that is insensitive to epithermal and fast neutrons. The thermal neutron imager counts neutrons with temporal and energy distributions very similar to the bare tube, but in addition provides directional information and automatic background subtraction.

\section{ACCELERATOR-RELATED DETECTOR ISSUES}

Accelerators produce intense radiation fields in their immediate vicinity, and detectors that are intended to be used near to them need to be designed to be impervious as possible to unwanted interference. Pulsed radio frequency signals from high voltage switching devices are very difficult to eliminate from sensitive preamplifier inputs. Very intense bursts of gamma rays can generate unwanted electronic signals by producing Compton electrons or photoelectrons at the internal surfaces of gas detectors and photomultipliers. Semiconductor devices can potentially suffer single event upsets and permanent radiation damage from intense beams of protons and neutrons. The signal of interest may be surpassed by that of background radiation, especially in the case of thermal neutrons that continue to diffuse from the floor and walls of an experimental area over a period of many seconds before they die away. If the accelerator is not completely enclosed by heavy shielding, there may be unintended radiation pathways by which the detectors are affected, making data interpretation difficult.

The BNL 2-dimensional position-sensitive ${ }^{3} \mathrm{He}$ wire chamber was originally designed for operation in continuous mode at a nuclear reactor. We modified the data acquisition system so that the data storage could be disabled during the short periods of interference by the accelerator and enabled by a gate signal with adjustable delayed start time and width. This modification prevented spurious electrical pulses in the preamplifiers from being counted as neutrons. However, it did not protect the preamplifiers from saturation during the gamma flash. During some experiments, depending on the distance and shielding between the accelerator and the detector, we observed that the analog signals were saturated for up to $1.5 \mathrm{~ms}$ after the accelerator pulses. Such a delay prevented the collection of $25 \mathrm{meV}$ neutrons with flight distances longer than $3.3 \mathrm{~m}$, which in most experiments would emphasize the room background scatter rather than the intended target. This phenomenon was found to be dependent on the multiplication of photoelectrons in the ${ }^{3} \mathrm{He}$ gas. By reducing the bias voltage on the anode wires, such that the voltage gain in the gas was reduced by a factor of 3 , we were able to operate the detector with a shorter dead time of the order of 200 microseconds. With lower gain, one would expect to compromise the spatial resolution, but we found that under these conditions the charge-division electronics still operated with good enough resolution that images were limited predominantly by the coded aperture mask design rather than the neutron position encoding.

Another potential limitation of using these detectors in the vicinity of accelerators is that an intense localized beam of ionizing radiation incident on the face of the wire chamber could cause long term changes in sensitivity. This type of degradation, known as aging, has happened before when a direct beam of thermal neutrons from the High Flux Beam Reactor was inadvertently allowed to irradiate a spot on the ${ }^{3} \mathrm{He}$ chamber for a few hours. The result was polymerization of the propane stopping gas (mixed with the ${ }^{3} \mathrm{He}$ ) to form an insulating layer on the wires. The detector had to be disassembled and cleaned with solvents in order to return it to normal operation. Since the localized neutron doses in our thermal neutron backscatter experiments are not expected to be comparable to those experienced at a reactor beam line, we do not expect a problem with degradation of the wire chamber unless much higher neutron fluxes are achieved by accelerators. 


\section{TYPICAL DATA}

The typical temporal distributions of prompt photoneutron counts from a $\mathrm{W}$ target surrounded with polyethylene for two detectors (PND and bare He-3) are shown in Fig. 1. The PND signal is high during the first $0.5 \mathrm{~ms}$ and then goes essentially to zero, indicating the thermalization time for the fast neutrons is less than $0.5 \mathrm{~ms}$. The count rates from the bare $\mathrm{He}-3$ tube continue to die away over $20 \mathrm{~ms}$ and is closely matched by the total count from the imager, most of which constitute neutrons scattered by the surroundings, giving a relatively uniform background to the image. The brightest pixel in the image, representing neutrons that travelled unscattered from the thermalizing material to the coded aperture, follows the theoretical model for thermal neutrons with a Maxwellian distribution of velocities traveling a distance of about 2 meters. The peak of the distribution at about $1 \mathrm{~ms}$, representing the mean thermal velocity of $25 \mathrm{meV}$ neutrons occurs at the same time for the PND, which was placed at the same distance from the target as the imager. After about 3 $\mathrm{ms}$, the imager is collecting predominantly scattered neutrons from distances further than the target, and the brightest pixel is in a random direction, no longer associated with the location of the target.

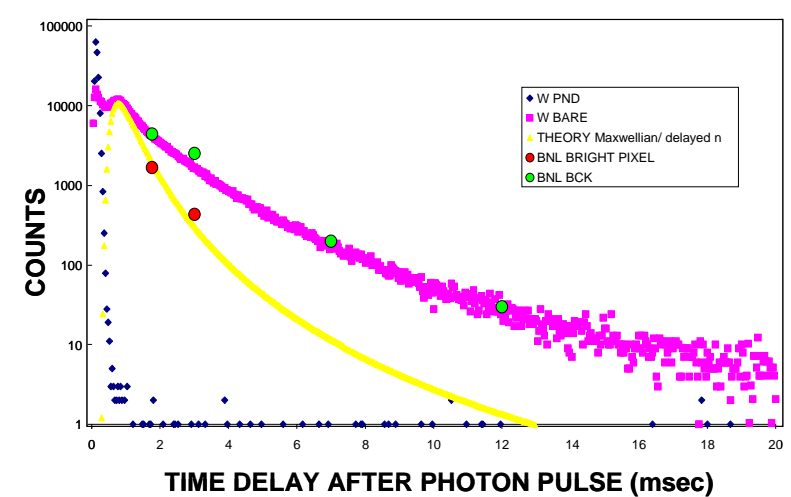

FIGURE 1. Neutron counts as a function of delay after photon pulse on a moderated $\mathrm{W}$ target. The counts in the unmoderated BNL detector are scaled to match the bare ${ }^{3} \mathrm{He}$ tube.

In the case of a fissionable material such as uranium (either enriched or depleted $\mathrm{U}$ can be induced to fission by photons above their photofission thresholds) the time response is markedly different, as shown in Fig. 2. The PND continues to register delayed neutrons from fission for several seconds after the excitation pulse. The brightest pixel continues to be measureable at delays greater than $1 \mathrm{~s}$, is associated with the target direction and is statistically significant relative to the uniform background.

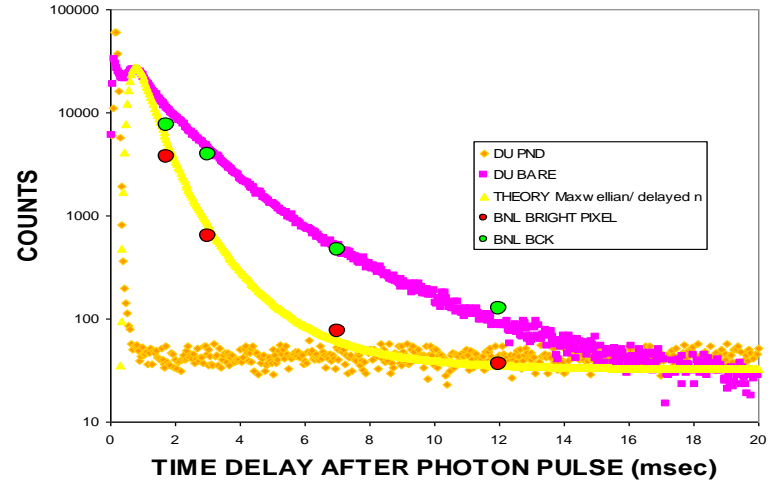

FIGURE 2. Neutron counts as a function of delay after photon pulse on a moderated ${ }^{238} \mathrm{U}$ target. The counts in the unmoderated BNL detector are scaled to match the bare ${ }^{3} \mathrm{He}$ tube.

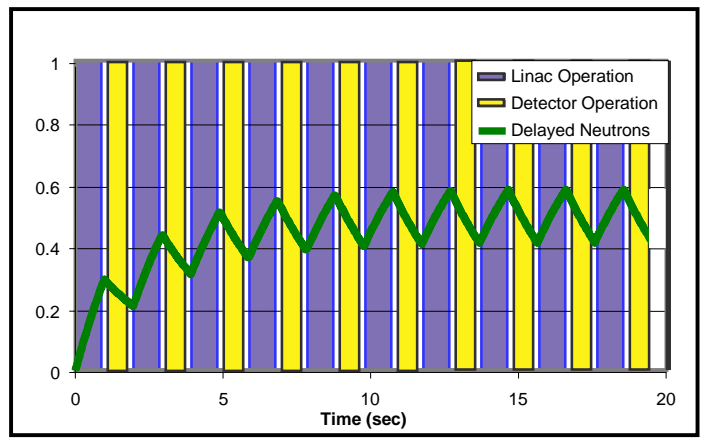

FIGURE 3. Schematic of build-up of flux of delayed neutrons after repetitive excitation with 1 -s beam pulses.

Fig. 3. shows a more effective way to configure the accelerator pulses and gate the detector in order to enhance the probability of detecting delayed neutrons. In this case, the beam is turned on for 1 second intervals interspersed with periods of 1 second when the beam is off. The population of excited states in the target that emit delayed neutrons is increased by repetitive excitation at a rate faster than the decay. The resulting thermal neutron image obtained at a distance of 2.4 meters from the target including about 1000 counts is shown in Fig. 4. The brightest pixel contains enough counts to be considered statistically significant beyond the Poisson distribution given by random counting, as illustrated in the histogram shown in Fig. 5. In cases where the source object is projected onto an area larger than one pixel, there may be several pixels with non-statistical counts. 


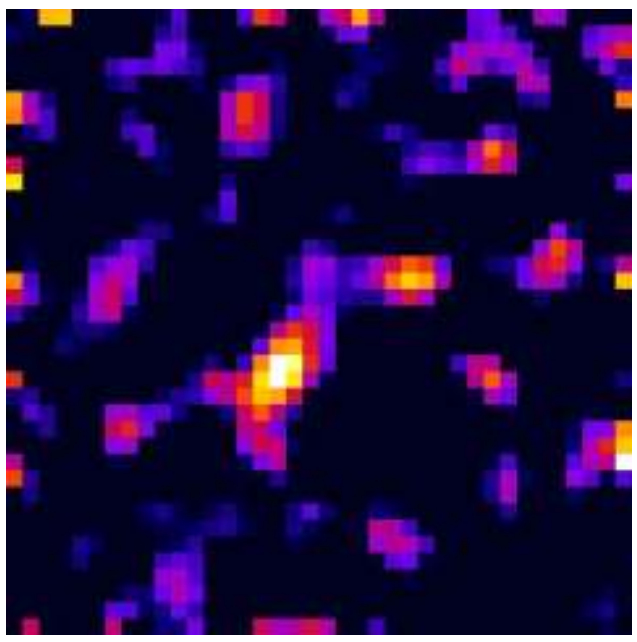

FIGURE 4. Thermal neutron image of moderated depleted uranium obtained with $\sim 1000$ delayed neutrons.

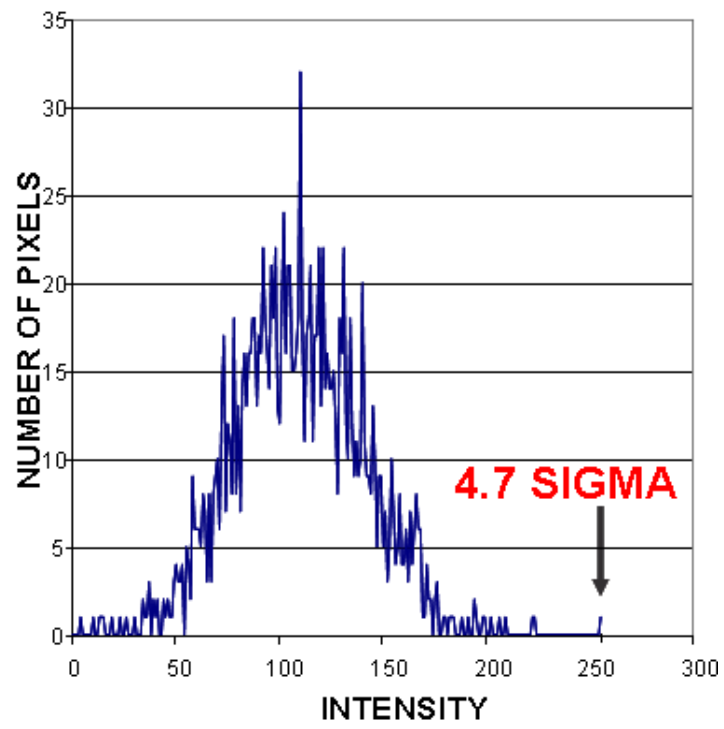

FIGURE 5. Distribution of intensities of pixels in the image data from Fig. 4, showing one pixel significantly brighter than the random distribution.

\section{CONCLUSIONS}

The BNL coded aperture thermal neutron camera can be used in the radiation environment of an electron linear accelerator and can help to distinguish a localized emitter of thermal neutrons from the diffuse background of neutrons.

\section{ACKNOWLEDGMENTS}

The authors are grateful to Dr. Alan Hunt and the staff of the Idaho Accelerator Center at Idaho State University for their assistance in performing some of these experiments.

This work was supported by U.S. Department of Energy, Office of Nonproliferation Research and Development. This manuscript has been authored by Brookhaven Science Associates, LLC under Contract No. DE-AC02-98CH1-886 with the U.S. Department of Energy. The United States Government retains, and the publisher, by accepting the article for publication, acknowledges, a world-wide license to publish or reproduce the published form of this manuscript, or allow others to do so, for the United States Government purposes.

\section{REFERENCES}

1. P. E. Vanier and L. Forman, "Advances in Imaging with Thermal Neutrons", Proceedings of the Institute of Nuclear Materials Management, 37th Annual Meeting, Naples, FL, (1996).

2. P.E.Vanier and L. Forman, "Forming Images with Thermal Neutrons", Invited Paper at the SPIE International Symposium on Optical Science and Technology, Conference 4784A, Hard X-rays, Gamma Rays and Particles, Seattle, WA, (2002).

3. Peter E. Vanier, Leon Forman, Stephen J. Hunter, Emma J. Harris, and Graham C. Smith, "Thermal Neutron Backscatter Imaging", Conference Record of IEEE Nuclear Science Symposium, Rome, (2004).

4. Victor Bom, Mostafa A. Ali, and Carel W.E. van Eijk, “ Land Mine Detection with Neutron Back Scattering Imaging using a Neutron Generator", IEEE Transactions on Nuclear Science, Vol 53, (2006) 356.

5. Klaus-Peter Ziock, Gus Caffrey, Alain Lebrun, Leon Forman, Peter Vanier and Jason Wharton, "Radiation Imaging of Dry Storage Casks for Spent Nuclear Fuel", Conference record of IEEE Nuclear Science Symposium, Puerto Rico, (2005).

6. Daren R. Norman, James L. Jones, Kevin J. Haskell, Peter E. Vanier and Leon Forman, "Active Nuclear Material Detection and Imaging", Conference Record of IEEE Nuclear Science Symposium, Puerto Rico, (2005). 